\title{
Producing a Scale-Invariant Spectrum of Perturbations in a Hagedorn Phase of String Cosmology
}

\section{Citation}

Nayeri, Ali, Robert H. Brandenberger, and Cumrun Vafa. 2006. “Producing a Scale-Invariant Spectrum of Perturbations in a Hagedorn Phase of String Cosmology." Physical Review Letters 97 (2). https://doi.org/10.1103/physrevlett.97.021302.

\section{Permanent link}

http://nrs.harvard.edu/urn-3:HUL.InstRepos:41384999

\section{Terms of Use}

This article was downloaded from Harvard University's DASH repository, and is made available under the terms and conditions applicable to Other Posted Material, as set forth at http:// nrs.harvard.edu/urn-3:HUL.InstRepos:dash.current.terms-of-use\#LAA

\section{Share Your Story}

The Harvard community has made this article openly available. Please share how this access benefits you. Submit a story. 


\title{
Producing a Scale-Invariant Spectrum of Perturbations in a Hagedorn Phase of String Cosmology
}

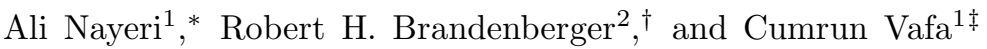 \\ 1 Jefferson Physical Laboratory, Harvard University, Cambridge, MA 02138, USA \\ 2 Department of Physics, McGill University, 3600 University Street, Montréal QC, H3A 2T8, Canada
}

(Dated: April 23, 2018)

\begin{abstract}
We study the generation of cosmological perturbations during the Hagedorn phase of string gas cosmology. Using tools of string thermodynamics we provide indications that it may be possible to obtain a nearly scale-invariant spectrum of cosmological fluctuations on scales which are of cosmological interest today. In our cosmological scenario, the early Hagedorn phase of string gas cosmology goes over smoothly into the radiation-dominated phase of standard cosmology, without having a period of cosmological inflation.
\end{abstract}

PACS numbers: $98.80 . \mathrm{Cq}$

Introduction. Since superstring theory contains many scalar fields, it is not unreasonable to study the possibility that a period of cosmological inflation might naturally arise from string theory (see e.g. 1, 2, 3] for recent review articles on this approach). Most of the work on trying to obtain inflation from string theory, however, is done in the framework of a low energy effective field theory motivated by string theory, and does not take into account new symmetries and new degrees of freedom of string theory which are hard to see at the level of an effective field theory (see also [4] for the restrictions that string theory can impose on the range of such a scalar field). In addition, the scalar field-driven inflationary paradigm, although phenomenologically very successful in terms of predicting an almost scale-invariant spectrum of adiabatic cosmological perturbations [5], suffers from several conceptual problems (see e.g. [6, 7] for discussions of these problems). In particular, there is an initial cosmological singularity. Thus, it is of great interest to explore the possibility of obtaining a new paradigm of early universe cosmology which is not based on scalar field-driven inflation but nevertheless predicts an almost scale-invariant spectrum of cosmological perturbations.

There is an early approach to string cosmology, now often called "string gas cosmology", which is based specifically on new symmetries (T-duality) and new degrees of freedom (string winding modes) of string theory [8] (see also [9]). Based on considerations of string thermodynamics, it was argued that string theory could provide a nonsingular cosmology. Going backwards in time, the universe contracts and the temperature grows. However, the temperature will not exceed the Hagedorn temperature. As radius of space approaches the self-dual radius (the string length), the pressure of the string gas will go to zero because of T-duality (the positive contribution to the pressure from momentum modes will cancel against the negative pressure from string winding modes). Using the background equations of motion from dilaton gravity, it follows that the evolution of the scale factor near the self-dual radius will be quasi-static [10]. Once the radius of space decreases below the self-dual radius, the string gas temperature will decrease, demonstrating that string gas cosmology will be non-singular. In addition, a mechanism was proposed [8] which might explain why, starting with all spatial dimensions of string scale, only three spatial dimensions can grow to be macroscopic. There has recently been quite a lot of work on further developing string gas cosmology (see e.g. 11, 12, 13, 14, 15) and [16, 17, 18] for recent reviews and comprehensive lists of references).

In string gas cosmology, it is assumed that the universe starts in a Hagedorn phase, a phase in which the universe is quasi-static and in thermal equilibrium at a temperature close to the Hagedorn temperature 19], the limiting temperature of perturbative string theory. As the universe slowly expands, heavy degrees of freedom gradually fall out of equilibrium. String winding modes keep all but three spatial dimensions compact [8] (see, however, 20, 21] for a critical view of this aspect of the scenario).

In this Letter, we will study the generation of cosmological fluctuations during the early Hagedorn phase of string gas cosmology using the tools of string statistical mechanics. Since this early phase is quasi-static, the Hubble radius $H^{-1}(t)$ is very large (infinite in the limit of the exactly static case). The approximation of thermodynamic equilibrium is justified on scales smaller than the Hubble radius. We demonstrate that, in this phase, a gas of closed strings induces a scale-invariant spectrum of scalar metric fluctuations on all scales smaller than the Hubble radius. Provided that the expansion of space is sufficiently slow, these scales will include all scales which are currently being probed by cosmological observations [30]. Provided that the spectrum in these fluctuations is not distorted at the time of the transition from the Hagedorn phase to the usual phase of radiation-domination of standard cosmology (which we argue is unlikely), it follows that string gas cosmology will lead - without invoking a period of inflation - to a scale-invariant spectrum of adiabatic curvature fluctuations. 


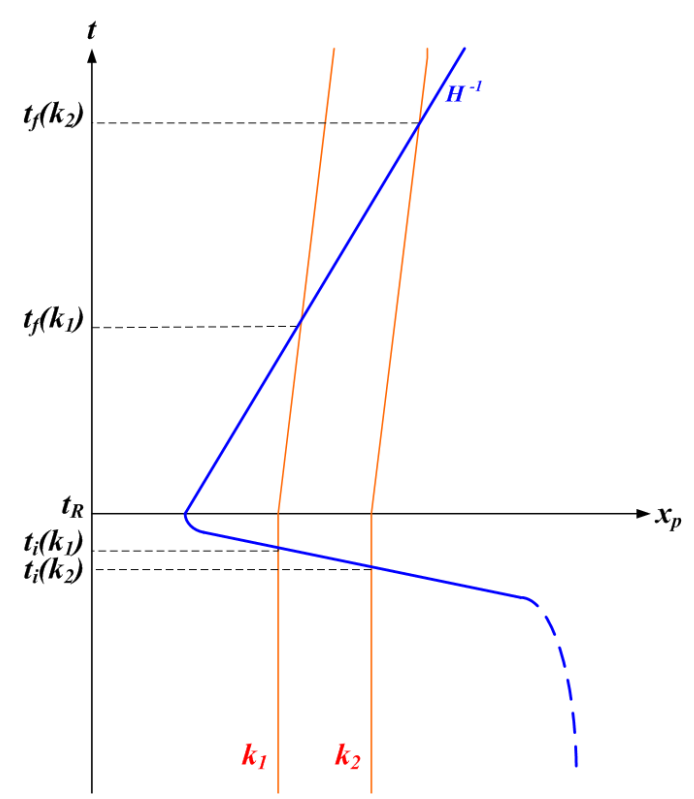

FIG. 1: Space-time diagram (sketch) showing the evolution of fixed comoving scales. The vertical axis is time, the horizontal axis is physical distance. The Hagedorn phase ends at the time $t_{R}$ and is followed by the radiation-dominated phase of standard cosmology. The blue curve represents the Hubble radius $H^{-1}$ which is cosmological during the quasistatic Hagedorn phase, shrinks abruptly to a microphysical scale at $t_{R}$ and then increases linearly in time for $t>t_{R}$. Fixed comoving scales (labeled by $k_{1}$ and $k_{2}$ ) which are currently probed in cosmological observations have wavelengths which are smaller than the Hubble radius during the Hagedorn phase. They exit the radius at times $t_{i}(k)$ just prior to $t_{R}$, and propagate with a wavelength larger than the Hubble radius until they reenter the Hubble radius at times $t_{f}(k)$.

Outline of the Analysis. Figure 1 presents a spacetime sketch of the history of the universe according to string gas cosmology. The initial phase $t<t_{R}$ is the Hagedorn phase during which space is quasi-static, and the temperature is close to the Hagedorn temperature $T_{H}$. For times $t>t_{R}$, the universe is assumed to be dominated by radiation as in standard cosmology. Since space-time is quasi-static during the Hagedorn phase, the Hubble radius is of cosmological scale. During the transition from the Hagedorn phase to the radiation-dominated phase, the Hubble radius shrinks dramatically to a microscopic scale given by

$$
H^{-1}\left(t_{R}\right) \sim \frac{M_{p l}}{T_{H}^{2}},
$$

where $M_{p l}$ is the four-dimensional Planck mass. In this paper, we assume that the radii of the extra spatial dimensions of string theory have already been stabilized. After $t_{R}$, the Hubble radius expands linearly in time 31].

In Figure 1, we also sketch the physical length for various fluctuation modes $k$ corresponding to fixed comoving wavenumber. The key feature is that the fluctuations are inside the Hubble radius during the Hagedorn phase. They exit the Hubble radius at times $t_{i}(k)$ which are close to the transition time $t_{R}$. The modes then reenter the Hubble radius at late times $t_{f}(k)$.

We want to calculate the amplitude of the metric fluctuations at late times in the phase of standard cosmology. In order to obtain agreement with current observations of cosmic microwave (CMB) anisotropies and large-scale structure, the spectrum of the metric fluctuations needs to be nearly scale-invariant and nearly adiabatic 29]. To compute these fluctuations, we will use the theory of linear cosmological perturbations about a four-dimensional homogeneous and isotropic cosmology (see [22] for a comprehensive review and 23] for a pedagogical introduction).

On scales larger than the Hubble radius, gravity dominates the dynamics and metric fluctuations play the leading role. We will calculate here the spectrum of scalar metric fluctuations, fluctuation modes which couple to the matter sources. In the absence of anisotropic stress, there is only one physical degree of freedom, namely the relativistic generalization of the Newtonian gravitational potential. In longitudinal gauge, the metric then takes the form

$$
d s^{2}=-(1+2 \Phi) d t^{2}+a(t)^{2}(1-2 \Phi) d \mathbf{x}^{2},
$$

where $t$ is physical time, $\mathbf{x}$ are the comoving spatial coordinates of the three large spatial dimensions, $a(t)$ is the cosmological scale factor and $\Phi(\mathbf{x}, t)$ represents the fluctuation mode.

On scales smaller than the Hubble radius, the gravitational potential $\Phi$ is determined by the matter fluctuations via the Einstein constraint equation (the relativistic generalization of the Poisson equation of Newtonian gravitational perturbation theory)

$$
\nabla^{2} \Phi=4 \pi G \delta \rho,
$$

where $\rho$ is the energy density.

Following the early analyses of the generation and evolution of cosmological perturbations in inflationary cosmology (see e.g. 24, 25, 26] for analyses in the spirit of what we do here), we will in the following calculate the power spectrum of mass fluctuations on sub-Hubble scales during the Hagedorn phase. We then use (3) to calculate the magnitude of the metric fluctuations $\Phi$ at the end of the Hagedorn phase (more specifically, at the time $t_{i}(k)$ when the scale labeled by $k$ exits the Hubble radius). As long as the equations of four space-time dimensional general relativistic cosmological perturbation theory apply, then $\Phi$ is conserved on super-Hubble scales as long as the equation of state of the background does not change significantly 32].

Assuming the validity of the arguments of the previous paragraphs, then the spectral index $n$ of the cosmological perturbations is determined by

$$
P_{\Phi}(k) \equiv k^{3}|\Phi(k)|^{2} \sim k^{n-1} .
$$


In the above, $\Phi(k)$ is the Fourier coefficient of $\Phi$ and $P_{X}$ denotes the dimensionless power spectrum of some quantity $X$. The value $n=1$ corresponds to a scaleinvariant spectrum.

Making use of the constraint equation (3), the dimensionless power spectrum $P_{\Phi}(k)$ of metric fluctuations can be expressed in terms of the mean square mass perturbations $\left\langle(\delta M)^{2}\right\rangle$ inside a sphere of radius $R=k^{-1}$ :

$$
P_{\Phi}(k)=16 \pi^{2} G^{2} k^{-4}\left\langle(\delta M)^{2}\right\rangle k^{6} .
$$

where the final factor $k^{-6}$ comes from converting density to mass. Thus, in order to establish a scale-invariant spectrum, we need to show that the mean square mass fluctuation $\left\langle(\delta M)^{2}\right\rangle$ scales as $k^{-2}$. In the following subsection we summarize this calculation. For details the reader is referred to a companion paper [27.

Computation of the Spectrum. Now we outline the calculation of the power spectrum of mass fluctuations. Starting point is the thermodynamic partition function

$$
Z(\beta)=\sum_{i} e^{-\beta E_{i}}
$$

where the summation runs over all states, $E_{i}$ is the energy of the state, and $\beta$ is the inverse temperature. The assumption here is the string coupling is sufficiently small, $g_{s} \ll 1$, and the local spacetime geometry is close to flat over the length scale of the finite size box of volume $V=R^{d}$. For that we consider a box of size $H^{-1}$ which consists of $N$ blocks of size $R$. In each block, $i$, the universe is homogenous and isotropic and filled with strings of energy $E_{i}$. In order to compute the mean square mass fluctuations in a region of radius $R=k^{-1}$, we apply string thermodynamics to a volume of that size. From (6) we obtain

$$
\left\langle(\delta M)^{2}\right\rangle=\left\langle E^{2}\right\rangle-\langle E\rangle^{2}=T^{2}\left(\frac{\partial\langle E\rangle}{\partial T}\right)_{V},
$$

where the angular brackets stand for thermodynamic averaging over the whole ensemble of the block universes, $\langle E\rangle$ is the ensemble average energy, and the subscript $V$ indicates that the partial derivative is taken at constant volume. In terms of the specific heat $C_{V} \equiv$ $(\partial\langle E\rangle / \partial T)_{V}$, the result becomes

$$
\left\langle(\delta M)^{2}\right\rangle=T^{2} C_{V}
$$

The specific heat of a gas of closed strings in a background space given by three large toroidal dimensions and six small compact dimensions is calculated in a companion paper [27], using the methods of [28]. The result, evaluated at temperatures close to the Hagedorn temperature, is

$$
C_{V} \simeq \frac{R^{2}}{\alpha^{\prime 3 / 2} T} \frac{1}{1-T / T_{H}}
$$

where $\alpha^{\prime}=\ell_{s}^{2}, \ell_{s}$ being the string length. Note that it was important to assume that all dimensions are compact in order to obtain positive specific heat. The mean square mass fluctuation can now immediately be read off from (7), and inserting into (5) leads to the final result for the spectrum of metric fluctuations:

$$
P_{\Phi} \sim 16 \pi^{2} G^{2} \alpha^{\prime-3 / 2} \frac{T}{1-T / T_{H}}
$$

From our final result (10) it follows that the spectrum of metric fluctuations is approximately scale-invariant, and that its amplitude is suppressed by the ratio $\left(\ell_{P l} / \ell_{s}\right)^{4}$, where $\ell_{P l}$ is the four dimensional Planck length. In order to obtain the observed amplitude of fluctuations [29], a hierarchy of lengths of the order of $10^{3}$ is required. This is consistent with our initial assumption that the string coupling constant should be really small since $\left(\ell_{P l} / \ell_{s}\right)=$ $g_{s} \sim 10^{-3} \ll 1$. Note that since for fixed value of $k$, the temperature $T$ which appears in the spectrum is to be evaluated at the time $t_{i}(k)$, a slight tilt of the spectrum towards a red spectrum is induced.

Discussion and Conclusions. In this Letter, we have studied the generation and evolution of cosmological fluctuations in a model of string gas cosmology in which an early quasi-static Hagedorn phase is followed by the radiation-dominated phase of standard cosmology, without an intervening period of inflation. Due to the fact that the Hubble radius during the Hagedorn phase is cosmological, it is possible to produce fluctuations using causal physics. Assuming thermal equilibrium on scales smaller than the Hubble radius, we have used string thermodynamics to study the amplitude of density fluctuations during the Hagedorn phase. The mean square mass fluctuations are determined by the specific heat of the string gas. To compute the perturbations on a physical length scale $R$, we apply string thermodynamics to a box of size $R$. Working under the assumption that all spatial dimensions are compact (but our three spatial dimensions are sufficiently large), the specific heat turns out to scale as $R^{2}$. This is an intrinsically stringy effect: in the case of point particle thermodynamics, the specific heat would scale as $R^{3}$. The $R^{2}$ scaling of the specific heat leads to a scale-invariant spectrum of metric fluctuations.

In order to compute the spectrum of metric fluctuations at late times, we have applied the usual general relativistic theory of cosmological perturbations. Whereas this is clearly justified for times $t>t_{R}$, its use at earlier times is doubtful. We have used the constraint equation coming from Einstein gravity to convert the matter fluctuations into metric perturbations immediately prior to $t_{R}$, when scales of cosmological interest today exit the Hubble radius. We have also assumed that the metric perturbation variable $\Phi$ does not change on super-Hubble scales during the transition between the Hagedorn phase and the radiation-dominated phase of standard cosmology. These assumptions are well justified in the con- 
text of the usual relativistic perturbation theory. However, the fact that the Hagedorn phase is described by a dilaton gravity background and not by a purely general relativistic background may lead to some modifications. However, since it was shown [10] that the usual radiation dominated phase with constant dilaton naturally emerges after the Hagedorn phase, there are good reasons to believe that the changes to our conclusions will be small.

Although our cosmological scenario provides a new mechanism for generating a scale-invariant spectrum of cosmological perturbations, it does not solve all of the problems which inflation solves. In particular, it does not solve the flatness problem. Without assuming that the three large spatial dimensions are much larger than the string scale, we do not obtain a universe which is sufficiently large today.

Our scenario may well be testable observationally. Taking into account the fact that the temperature $T$ evaluated at the time $t_{i}(k)$ when the scale $k$ exits the Hubble radius depends slightly on $k$, the formula (10) leads to a calculable deviation of the spectrum from exact scale-invariance. Since $T\left(t_{i}(k)\right)$ is decreasing as $k$ increases, a slightly red spectrum is predicted. Since the equation of state does not change by orders of magnitude during the transition between the initial phase and the radiation-dominated phase as it does in inflationary cosmology, the spectrum of tensor modes is not expected to be suppressed compared to that of scalar modes. Hence, a large ratio of tensor to scalar fluctuations might be a specific prediction of our model. This issue deserves further attention.

Acknowledgments. The work of A.N. and C.V. is supported in part by NSF grant PHY-0244821 and DMS0244464. R.B. wishes to thank the Harvard High Energy Theory group for its hospitality during visits when this work was initiated and completed. The work of R.B. is supported by funds from McGill University, by an NSERC Discovery Grant and by the Canada Research Chair program.

* Electronic address: nayeri@schwinger.harvard.edu

† Electronic address: rhb@karabine.physics.mcgill.ca

¥ Electronic address: vafa@string.harvard.edu

[1] A. Linde, eConf C040802, L024 arXiv:hep-th/0503195.

[2] C. P. Burgess, Pramana 63, 1269 arXiv:hep-th/0408037.

[3] J. M. Cline, arXiv:hep-th/0501179

[4] C. Vafa, arXiv:hep-th/0509212

[5] V. F. Mukhanov and G. V. Chibisov, JETP Lett. 33, 532 (1981) [Pisma Zh. Eksp. Teor. Fiz. 33, 549 (1981)]; V. N. Lukash, Sov. Phys. JETP 52, 807 (1980) [Zh. Eksp. Teor. Fiz. 79, (1980)].

[6] R. H. Brandenberger, arXiv:hep-ph/9910410.

[7] R. H. Brandenberger, arXiv:hep-th/0509076
[8] R. H. Brandenberger and C. Vafa, Nucl. Phys. B 316, 391 (1989).

[9] J. Kripfganz and H. Perlt, Class. Quant. Grav. 5, 453 (1988).

[10] A. A. Tseytlin and C. Vafa, Nucl. Phys. B 372, 443 (1992) arXiv:hep-th/9109048.

[11] S. Alexander, R. H. Brandenberger and D. Easson, Phys. Rev. D 62, 103509 (2000) arXiv:hep-th/0005212.

[12] S. Watson and R. Brandenberger, JCAP 0311, 008 (2003) arXiv:hep-th/0307044.

[13] S. P. Patil and R. Brandenberger, Phys. Rev. D 71, 103522 (2005) arXiv:hep-th/0401037.

[14] S. P. Patil and R. H. Brandenberger, arXiv:hep-th/0502069

[15] R. Brandenberger, Y. K. Cheung and S. Watson, arXiv:hep-th/0501032

[16] R. Brandenberger, arXiv:hep-th/0509099, Challenges for String Gas Cosmology to appear in the proceedings of the 59th Yamada Conference "Inflating Horizon of Particle Astrophysics and Cosmology" (University of Tokyo, Tokyo, Japan, June 20 - June 24, 2005).

[17] T. Battefeld and S. Watson, arXiv:hep-th/0510022

[18] R. Brandenberger, Moduli Stabilization in String Gas Cosmology to appear in the proceedings of YKIS 2005 (Yukawa Institute for Theoretical Physics, Kyoto, Japan, June 27 - July 1, 2005). arXiv:hep-th/0509159

[19] R. Hagedorn, Nuovo Cim. Suppl. 3, 147 (1965).

[20] R. Easther, B. R. Greene, M. G. Jackson and D. Kabat, JCAP 0502, 009 (2005) arXiv:hep-th/0409121.

[21] R. Danos, A. R. Frey and A. Mazumdar, Phys. Rev. D 70, 106010 (2004) arXiv:hep-th/0409162.

[22] V. F. Mukhanov, H. A. Feldman and R. H. Brandenberger, Phys. Rept. 215, 203 (1992).

[23] R. H. Brandenberger, Lect. Notes Phys. 646, 127 (2004) arXiv:hep-th/0306071.

[24] J. M. Bardeen, P. J. Steinhardt and M. S. Turner, Phys. Rev. D 28, 679 (1983).

[25] R. H. Brandenberger and R. Kahn, Phys. Rev. D 29, 2172 (1984).

[26] R. H. Brandenberger, Nucl. Phys. B 245, 328 (1984).

[27] A. Nayeri, companion paper.

[28] N. Deo, S. Jain and C. I. Tan, Phys. Lett. B 220, 125 (1989); N. Deo, S. Jain and C. I. Tan, Phys. Rev. D 40, 2626 (1989); N. Deo, S. Jain, O. Narayan and C. I. Tan, Phys. Rev. D 45, 3641 (1992).

[29] D. N. Spergel et al. [WMAP Collaboration], Astrophys. J. Suppl. 148, 175 (2003) arXiv:astro-ph/0302209.

[30] We are also assuming by fiat that the three "large" dimensions of space are sufficiently large during the Hagedorn phase such that a universe encompassing the presently observed Hubble radius can emerge from it during the usual expansion history of standard cosmology.

[31] In order to explain the current size of the universe, the radius of the three large dimensions must be at least $10^{23}$ times the string scale at the Hagedorn temperature - a quantification of the flatness (or size) problem which our scenario does not resolve.

[32] The use of ordinary general relativistic cosmological perturbation theory is certainly justified after the end of the Hagedorn phase, but not necessarily in the time interval between $t_{i}(k)$ and $t_{R}$. We intend to come back to a careful study of the evolution of fluctuations during this time interval in a followup paper. 